\title{
Derivation of Pressure Distribution Models for Horizontal Well Using Source Function
}

\section{*1OLORO, JO; ${ }^{2}$ ADEWOLE, ES}

\author{
${ }^{1}$ Department of Chemical and Petroleum Engineering, Delta State University, Abraka, Nigeria \\ ${ }^{2}$ Department of Petroleum Engineering, University of Benin, Nigeria \\ *Corresponding Author Email: joloroeng@yahoo.com
}

\begin{abstract}
In oil and gas operation, horizontal well technology has become an important technique because of its numerous advantages. Despite these advantages, determination of pressure distribution expression is a problem because it involve 3D transient-flow that need to be solved. In this work, ten (10) models for pressure distribution for horizontal well under different boundary variation were derived following these steps for each of the models:(i) choosing a boundary condition for each axis(ii)selecting the appropriate source function for each axis and (iii)applying Newman product rule to arrive at the pressure expression. In conclusion, the following were observed.(a) along the $x-$ axis, seven of the models have infinite-slab sources in infinite-slab sources(b) five of the models derived, along the yaxis they have infinite-plane sources in infinite-slab sources while for the remaining five along the y- axis they have infinite sources(c) along z-axis, six of the models have infinite-plane sources in infinite-slab sources while four of the remaining one have infinite sources(d) Three types of boundaries are observed:(i) Completely bounded boundaries (ii) Mixed boundaries (iii) infinite-acting boundaries(e)Three instantaneous source functions are involved in the pressure distribution expression.
\end{abstract}

\begin{tabular}{|c|c|c|c|}
\hline \multicolumn{2}{|c|}{ NOMENCLATURE } & $y_{e}=$ & distance to the external boundary in $y$-direction \\
\hline$h_{D}=$ & dimensionless height & $\mathrm{x}_{\mathrm{e}}=$ & distance to the external boundary in $x$-directic \\
\hline$x_{D}=$ & arbitrary dimensionless distance along the $\mathrm{x}$ axis & $\mathrm{z}_{\mathrm{e}}=$ & distance to the external boundary in $z$-directic \\
\hline $\mathrm{y}_{\mathrm{D}}=$ & arbitrary dimensionless distance along the y axis & $\mathrm{z}_{\mathrm{w}}=$ & Well coordinate in z-direction \\
\hline $\mathrm{Z}_{\mathrm{D}}=$ & arbitrary dimensionless distance along the $\mathrm{z}$ axis & $\mathrm{y}_{\mathrm{w}}=$ & Well coordinate in y-direction \\
\hline $\mathbf{\tau}=$ & dummy integration dimensionless time variable & $\mathrm{x}_{\mathrm{w}}=$ & Well coordinate in $\mathrm{x}$-direction \\
\hline$\eta=$ & diffusivity constant 1 & $\mathrm{t}=$ & Time \\
\hline
\end{tabular}

DOI: https://dx.doi.org/10.4314/jasem.v23i4.1

Copyright: Copyright (C) 2019 Oloro and Adewole. This is an open access article distributed under the Creative Commons Attribution License (CCL), which permits unrestricted use, distribution, and reproduction in any medium, provided the original work is properly cited.

Dates: Received: 07 February 2019; Revised: 19 March 2019; Accepted 02 April 2019

Keywords: Source function, Horizontal well, Reservoir, Boundary

\section{INTRODUCTION}

In oil and gas exploitation, horizontal well technique has turn out to be an important practice because of its capability to produce with a higher flow rate at a given reservoir pressure (Ohaegbulam, et al., 2017), Determination of pressure distribution expression is a problem because it involve 3D transient-flow, that need to be solved (Larry, 2006). Pressure distribution expressions are used to (1) develop well test analysis procedure, (2) estimate reservoir pressures, (3) estimate wellbore flowing pressure, (4) determine and forecast the performance of recovery processes. In this work, we will be using source functions to derive models for pressure distribution for horizontal well under different boundary variation. Gringarten and Ramey presented the similar forms of the instantaneous linear source and the time ranges for this approximation to be valid (Gringarten, et al., 1973). Adewole and Olafuyi also use source to derive different pressure expression for two layer reservoir in an A shaped architecture. One of their results shows that all the sources have infinite boundary at the bottom (Adewole, et al., 2010). Eiroboyi and Wilkie presented comparative study of horizontal well and vertical well both subjected by edge water using source function and their results shows that for all reservoir geometry, the horizontal well will withstand longer period of the radial flow than vertical well given infinite conductivity condition (Eiroboyi, et $a l ., 2017)$. Jacob Overgaard work on how to use Source Function (SF) for the reconstruction of the electron density (Jacob Overgaard, 2016). Chawla and Kirby used source function method for generation of waves on currents in Boussinesq models (Chawla, et al., 2000). The objective of this work is to derive some models that be able to be used to determine pressure distribution for horizontal well.

\section{MATERIALS AND METHODS}


The following steps are taken in deriving each of the models.

1. Chose a boundary condition for each axis.

2. Select the appropriate source function for each axis (Gringarten, et al., 1973).

3. Apply Newman product rule to arrive at the pressure expression.

For the purpose of deriving pressure distribution expressions, the boundaries of the reservoir will be varied as either completely, partially sealed, completely or partially constant-pressured, or mixed and constant-pressured. Figure 1 shows complete bounded reservoir. The reservoir has length $x_{e}$ in $x-$ direction, length $y_{e}$ in $y$-direction and length $z_{e}$ in $z^{-}$ direction. It has a horizontal well locations $\mathrm{x}_{\mathrm{w}, \mathrm{y}_{\mathrm{w}} \text { and }}$ $\mathrm{z}_{\mathrm{w}}$ in $\mathrm{x}, \mathrm{y}$ and $\mathrm{z}$ directions respectively. All horizontal well dimensions originate from the Centre of the well with coordinate $\left(x_{w}, y_{w} L / 2\right.$, and $\left.z_{w}\right)$. The source functions are selected from basic instantaneous source functions table for $\mathrm{x}, \mathrm{y}$ and $\mathrm{z}$ axis's given as viii(x), $\mathrm{v}(\mathrm{y}), \mathrm{v}(\mathrm{z})$.Equation 1,2 and 3 are the source functions for the three axes in dimensionless form.

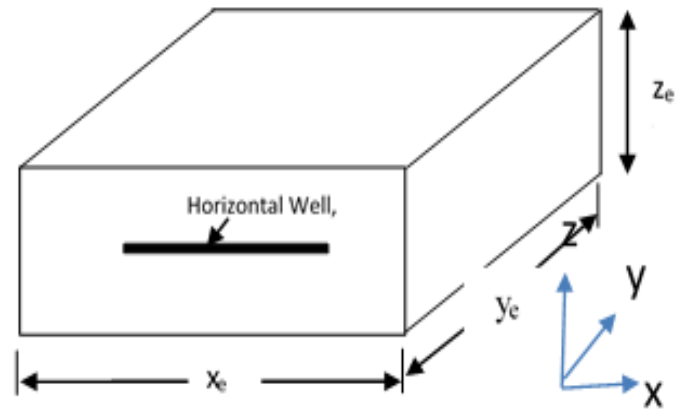

Fig 1: 3D Reservoir Model

A Completely Bounded Reservoir: Using equation 4 known as Newman product method, .putting equation 1, 2 and 3 in 4 we have equation 5. Equation 5 is the pressure distribution for completely bounded reservoir.

$$
\begin{aligned}
& S\left(x_{D}, t_{D}\right)=\frac{2}{X_{e D}}\left\{1+\frac{2 X_{e D}}{\pi} \sum_{n=1}^{\infty} \frac{1}{n} \exp \left[-\frac{n^{2} X_{D}{ }^{2} t_{D}}{X_{e D}{ }^{2}}\right] \sin \frac{n \pi}{x_{e D}} \cos \frac{n \pi x_{w D}}{X_{e D}} \cos \frac{n \pi x_{D}}{X_{e D}}\right\} \\
& S\left(y_{D}, t_{D}\right)=\frac{1}{y_{D}}\left\{1+2 \sum_{n=1}^{\infty} \exp \left[-\frac{m^{2} \pi^{2} t_{D}}{y_{D}}\right] \cos \frac{m \pi y_{w D}}{y_{D}} \cos \frac{m \pi y_{D}}{y_{D}}\right\} \\
& S\left(z_{D}, t_{D}\right)=\frac{1}{h_{D}}\left\{1+2 \sum_{n=1}^{\infty} \exp \left[-\frac{l^{2} \pi^{2} t_{D}}{h_{D}}\right] \cos \frac{l \pi z_{w D}}{h_{D}} \cos \frac{l \pi h_{D}}{h_{D}}\right\}
\end{aligned}
$$

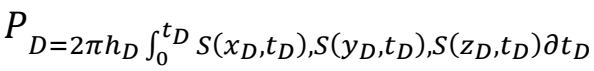

$$
\begin{aligned}
& \mathrm{P}=\frac{2}{\mathrm{X}_{\mathrm{eD}}}\left\{1+\frac{2 \mathrm{X}_{\mathrm{eD}}}{\pi} \sum_{\mathrm{n}=1}^{\infty} \frac{1}{\mathrm{n}} \exp \left[-\frac{\mathrm{n}^{2} \mathrm{X}_{\mathrm{D}}{ }^{2} \mathrm{t}_{\mathrm{D}}}{\mathrm{X}_{\mathrm{eD}}{ }^{2}}\right] \sin \frac{\mathrm{n} \pi}{\mathrm{X}_{\mathrm{eD}}} \cos \frac{\mathrm{n} \pi \mathrm{x}_{\mathrm{wD}}}{\mathrm{X}_{\mathrm{eD}}} \cos \frac{\mathrm{n} \pi \mathrm{x}_{\mathrm{D}}}{\mathrm{X}_{\mathrm{eD}}}\right\} * \\
& \frac{1}{y_{D}}\left\{1+2 \sum_{n=1}^{\infty} \exp \left[-\frac{m^{2} \pi^{2} t_{D}}{y_{D}}\right] \cos \frac{m \pi y_{w D}}{y_{D}} \cos \frac{m \pi y_{D}}{y_{D}}\right\} * \\
& \frac{1}{h_{D}}\left\{1+2 \sum_{n=1}^{\infty} \exp \left[-\frac{l^{2} \pi^{2} t_{D}}{h_{D}}\right] \cos \frac{l \pi z_{W D}}{h_{D}} \cos \frac{l \pi h_{D}}{h_{D}}\right\}
\end{aligned}
$$

Only $x$-axis is sealed Figure 2 shows reservoir of length $x_{e}$ in $x$-direction, length $y$ in $y$-direction and length $\mathrm{z}$ in $\mathrm{z}$-direction. It has a horizontal well locations $\mathrm{x}_{\mathrm{w}}, \mathrm{y}_{\mathrm{w}}$ and $\mathrm{z}_{\mathrm{w}}$ in $\mathrm{x}, \mathrm{y}$ and $\mathrm{z}$ directions respectively. Only the boundaries coinciding with the $\mathrm{x}$-axis are sealed. The source functions are selected from basic instantaneous source functions table for $\mathrm{x}, \mathrm{y}$ and $\mathrm{z}$ axis's ,given as viii(x), i(y), i(z).Equation 6,7 and 8 are the source functions for the three axes in dimensionless form.

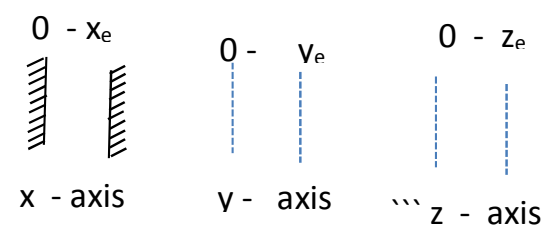

Fig 2: Only $x$-axis so suanu 


$$
\begin{gathered}
S\left(x_{D}, t_{D}\right)=\frac{2}{X_{e D}}\left\{1+\frac{2 X_{e D}}{\pi} \sum_{n=1}^{\infty} \frac{1}{n} \exp \left[-\frac{n^{2} X_{D}^{2} t_{D}}{X_{e D}^{2}}\right] \sin \frac{n \pi}{x_{e D}} \cos \frac{n \pi x_{w D}}{X_{e D}} \cos \frac{n \pi x_{D}}{X_{e D}}\right\} \\
S\left(y_{D}, t_{D}\right)=\frac{1}{2 \sqrt{\pi t_{D}}} \exp \left[-\frac{\left(y_{D}-y_{w D}\right)^{2}}{4 t_{D}}\right] \\
S\left(\mathrm{z}_{\mathrm{D}}, \mathrm{t}_{\mathrm{D}}\right)=\frac{1}{2 \sqrt{\pi \mathrm{t}_{\mathrm{D}}}} \exp \left[-\frac{\left(\mathrm{Z}_{\mathrm{D}}-\mathrm{Z}_{\mathrm{wD}}\right)^{2}}{4 \mathrm{t}_{\mathrm{D}}}\right]
\end{gathered}
$$

Putting equation 6,7 and 8 in 4 we have Equation 9 as the pressure distribution for reservoir sealed only at xaxis.

$$
\begin{aligned}
P_{D=} & \frac{2}{X_{e D}}\left\{1+\frac{2 X_{e D}}{\pi} \sum_{n=1}^{\infty} \frac{1}{n} \exp \left[-\frac{n^{2} X_{D}{ }^{2} t_{D}}{X_{e D}{ }^{2}}\right] \sin \frac{n \pi}{x_{e D}} \cos \frac{n \pi x_{w D}}{X_{e D}} \cos \frac{n \pi x_{D}}{X_{e D}}\right\} * \\
& \frac{1}{2 \sqrt{\pi t_{D}}} \exp \left[-\frac{\left(y_{D}-y_{W D}\right)^{2}}{4 t_{D}}\right] * \frac{1}{2 \sqrt{\pi t_{D}}} \exp \left[-\frac{\left(\mathrm{z}_{\mathrm{D}}-\mathrm{Z}_{\mathrm{WD}}\right)^{2}}{4 \mathrm{t}_{\mathrm{D}}}\right]
\end{aligned}
$$

Reservoir subject to Edge water and sealed at $y$ and $z$ axes: Figure 3 is a reservoir of length $\mathrm{x}_{\mathrm{e}}$ in $\mathrm{x}-$ direction, length $y_{e}$ in $y$-direction and length $z_{e}$ in $z-$ direction subjected by edge water. It has a horizontal

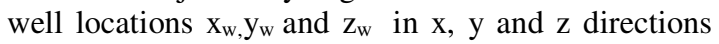
respectively. The source functions are selected from basic instantaneous source functions table for $\mathrm{x}, \mathrm{y}$ and $\mathrm{z}$ axis's given as $\mathrm{xi}(\mathrm{x}), \mathrm{v}(\mathrm{y}), \mathrm{v}(\mathrm{z})$.Equation 10,11 and 12 are the source functions the three axes in dimensionless form of equation.

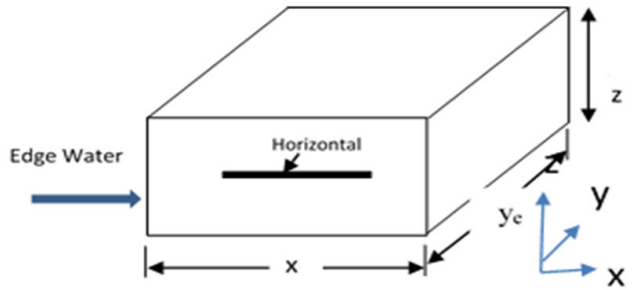

Fig 3: Reservoir subject to Edge water and sealed at $y$ and $z$ axes

$$
\begin{aligned}
& S\left(x_{D}, t_{D}\right)=\frac{8}{\pi} \sum_{n=1}^{\infty} \frac{1}{2 n-1} \exp \left[-\frac{(2 n-1)^{2} \pi^{2} t_{D}}{x_{e D}{ }^{2}}\right] \cos \frac{(2 n-1) \pi x_{D}}{2 x_{e D}} \\
& * \sin \frac{(2 n-1) \pi x_{D}}{x_{e D}} \sin \frac{(2 n-1) \pi x_{W D}}{x_{e D}} \\
& S\left(y_{D}, t_{D}\right)=\frac{1}{y_{D}}\left\{1+2 \sum_{n=1}^{\infty} \exp \left[-\frac{m^{2} \pi^{2} t_{D}}{y_{D}}\right] \cos \frac{m \pi y_{w D}}{y_{D}} \cos \frac{m \pi y_{D}}{y_{D}}\right\}
\end{aligned}
$$

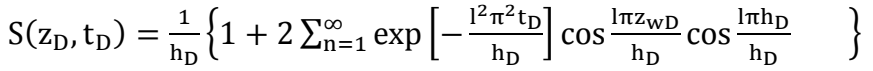

Putting equation 10, 11 and 12 in 4 we have Equation 13 as the pressure distribution for reservoir sealed at $\mathrm{x}$ and $\mathrm{y}$ axes and subjected by edge water.

$$
\begin{aligned}
& P_{D}=\frac{8}{\pi} \sum_{n=1}^{\infty} \frac{1}{2 n-1} \exp \left[-\frac{(2 n-1)^{2} \pi^{2} t_{D}}{x_{e D}^{2}}\right] \cos \frac{(2 n-1) \pi x_{D}}{2 x_{e D}} \sin \frac{(2 n-1) \pi x_{D}}{x_{e D}} \sin \frac{(2 n-1) \pi x_{W D}}{x_{e D}} \\
& * \frac{1}{y_{D}}\left\{1+2 \sum_{n=1}^{\infty} \exp \left[-\frac{m^{2} \pi^{2} t_{D}}{y_{D}}\right] \cos \frac{m \pi y_{w D}}{y_{D}} \cos \frac{m \pi y_{D}}{y_{D}}\right\} \\
& * \frac{1}{h_{D}}\left\{1+2 \sum_{n=1}^{\infty} \exp \left[-\frac{l^{2} \pi^{2} t_{D}}{h_{D}}\right] \cos \frac{l \pi z_{w D}}{h_{D}} \cos \frac{l \pi h_{D}}{h_{D}}\right\}
\end{aligned}
$$

Reservoir Subject to Bottom Water and Sealed at $X$ and $Y$ Axes: Figure 4 shows the reservoir in 3D of length $\mathrm{x}_{\mathrm{e}}$ in $\mathrm{x}$-direction, length $\mathrm{y}_{\mathrm{e}}$ in $\mathrm{y}$-direction and length $z_{\mathrm{e}}$ in $\mathrm{z}$-direction subjected $\mathrm{b}$ bottom water. It has a horizontal well locations $\mathrm{x}_{\mathrm{w}}, \mathrm{y}_{\mathrm{w}}$ and $\mathrm{z}_{\mathrm{w}}$ in $\mathrm{x}, \mathrm{y}$ and $\mathrm{z}$ directions respectively. The source functions are selected from basic instantaneous source functions table for $\mathrm{x}, \mathrm{y}$ and $\mathrm{z}$ axis's given as viii(x), $\mathrm{v}(\mathrm{y})$, vii(z)b. Equation 14,15 and 16 are the source functions for the three axes in dimensionless form.

Top Gas Cap and Bottom Water drive: Figure 5 represent the $3 \mathrm{D}$ of the reservoir model and axial description of the reservoir of length $x_{e}$ in $x$ direction, length $y_{e}$ in $y$-direction and length $z$ in $z$ - 
direction subjected by Top Gas cap and bottom water. It has a horizontal well locations $\mathrm{x}_{\mathrm{w},} \mathrm{y}_{\mathrm{w}}$ and $\mathrm{z}_{\mathrm{w}}$ in $\mathrm{x}, \mathrm{y}$ and $\mathrm{z}$ directions respectively. The source functions are selected from basic instantaneous source functions table for $\mathrm{x}, \mathrm{y}$ and $\mathrm{z}$ axis's given as viii( $\mathrm{x}), \mathrm{v}(\mathrm{y})$, vii(z)b. Equation 18,19 and 20 are the source functions the three axes in dimensionless form.
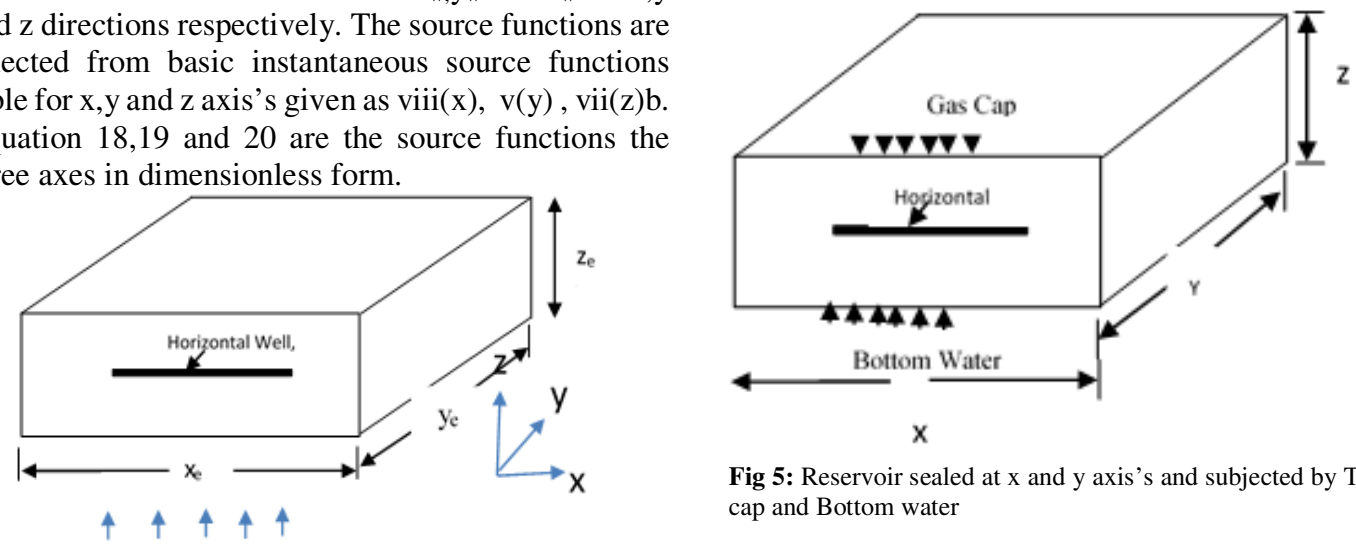

Fig 5: Reservoir sealed at $x$ and $y$ axis's and subjected by Top Gas cap and Bottom water

Bottom Water

Fig 4: Bottom water and sealed at $x$ and $y$ axes

$$
\begin{gathered}
S\left(x_{D}, t_{D}\right)=\frac{2}{X_{e D}}\left\{1+\frac{2 x_{e D}}{\pi} \sum_{n=1}^{\infty} \frac{1}{n} \exp \left[-\frac{n^{2} X_{D}{ }^{2} t_{D}}{X_{e D}{ }^{2}}\right] \sin \frac{n \pi}{x_{e D}} \cos \frac{n \pi x_{w D}}{X_{e D}} \cos \frac{n \pi x_{D}}{X_{e D}}\right\} \\
S\left(y_{D}, t_{D}\right)=\frac{1}{y_{D}}\left\{1+2 \sum_{n=1}^{\infty} \exp \left[-\frac{m^{2} \pi^{2} t_{D}}{y_{D}}\right] \cos \frac{m \pi y_{w D}}{y_{D}} \cos \frac{m \pi y_{D}}{y_{D}}\right\} \\
S\left(z_{D}, t_{D}\right)=\frac{1}{h_{D}}\left\{1+2 \sum_{n=1}^{\infty} \exp \left[-\frac{l^{2} \pi^{2} t_{D}}{h_{D}}\right] \cos \frac{l \pi z_{w D}}{h_{D}} \cos \frac{l \pi h_{D}}{h_{D}}\right\}
\end{gathered}
$$

Putting equation 14,15 and 16 in 4 we have Equation 17 as the pressure distribution for reservoir sealed at $\mathrm{x}$ and y axis's and subjected by bottom water.

$$
\begin{aligned}
& P_{D}=2 \pi h_{D} \int_{0}^{t_{D}} \frac{2}{\mathrm{X}_{\mathrm{eD}}}\left\{\begin{array}{c}
1+\frac{2 \mathrm{X}_{\mathrm{eD}}}{\pi} \sum_{\mathrm{n}=1}^{\infty} \frac{1}{\mathrm{n}} \exp \left[-\frac{\left.\mathrm{n}^{2} \mathrm{X}_{\mathrm{D}}^{2} \mathrm{t}_{\mathrm{D}}\right] \sin \frac{\mathrm{n} \pi}{\mathrm{X}_{\mathrm{eD}}{ }^{2}} \cos \frac{\mathrm{n} \pi \mathrm{x}_{\mathrm{WD}}}{\mathrm{X}_{\mathrm{eD}}} \cos \frac{\mathrm{n} \pi \mathrm{x}_{\mathrm{D}}}{\mathrm{X}_{\mathrm{eD}}}}{\mathrm{x}_{\mathrm{D}}}\left\{1+2 \sum_{n=1}^{\infty} \exp \left[-\frac{m^{2} \pi^{2} t_{D}}{y_{D}}\right] \cos \frac{m \pi y_{w D}}{y_{D}} \cos \frac{m \pi y_{D}}{y_{D}}\right\}\right. \\
* \frac{1}{h_{D}}\left\{1+2 \sum_{n=1}^{\infty} \exp \left[-\frac{l^{2} \pi^{2} t_{D}}{h_{D}}\right] \cos \frac{l \pi z_{w D}}{h_{D}} \cos \frac{l \pi h_{D}}{h_{D}}\right\}
\end{array}\right\} \partial t_{D} \\
& S\left(x_{D}, t_{D}\right)=\frac{2}{\mathrm{x}_{\mathrm{eD}}}\left\{1+\frac{2 \mathrm{x}_{\mathrm{eD}}}{\pi} \sum_{\mathrm{n}=1}^{\infty} \frac{1}{\mathrm{n}} \exp \left[-\frac{\mathrm{n}^{2} \mathrm{x}_{\mathrm{D}}{ }^{2} \mathrm{t}_{\mathrm{D}}}{\mathrm{x}_{\mathrm{eD}}{ }^{2}}\right] \sin \frac{\mathrm{n} \pi}{\mathrm{x}_{\mathrm{eD}}} \cos \frac{{\mathrm{n} \pi \mathrm{x}_{\mathrm{wD}}}_{\mathrm{x}_{\mathrm{eD}}}}{\cos } \frac{\mathrm{n} \pi \mathrm{x}_{\mathrm{D}}}{\mathrm{x}_{\mathrm{eD}}}\right\} \\
& S\left(y_{D}, t_{D}\right)=\frac{1}{y_{D}}\left\{1+2 \sum_{n=1}^{\infty} \exp \left[-\frac{m^{2} \pi^{2} t_{D}}{y_{D}}\right] \cos \frac{m \pi y_{w D}}{y_{D}} \cos \frac{m \pi y_{D}}{y_{D}}\right\} \\
& S\left(z_{D}, t_{D}\right)=\frac{1}{h_{D}}\left\{1+2 \sum_{n=1}^{\infty} \exp \left[-\frac{l^{2} \pi^{2} t_{D}}{h_{D}}\right] \sin \frac{l \pi z_{D}}{h_{D}} \sin \frac{l \pi z_{w D}}{h_{D}}\right\}
\end{aligned}
$$

Putting equation 18, 19 and 20 in 4 we have Equation 21 as the pressure distribution for reservoir sealed at $\mathrm{x}$ and y axes and subjected by top Gas cap and bottom water. This Model (Equation 21) Reservoir with underlain water and gas production.

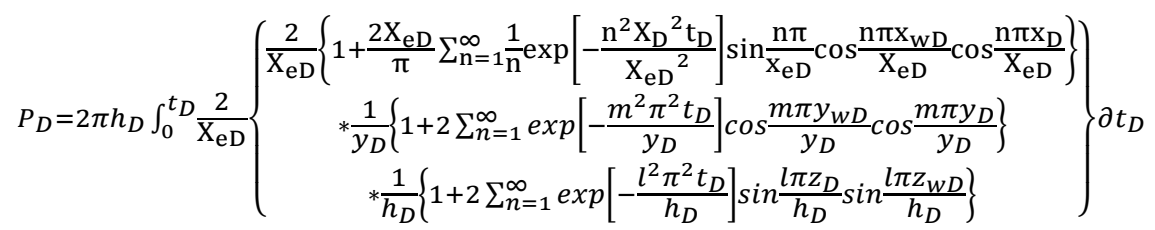


All boundaries are Infinite acting: Figure 6 is a reservoir with all boundaries infinite-acting. It has a horizontal

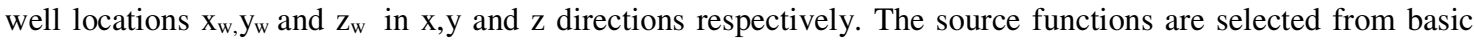
instantaneous source functions table for $\mathrm{x}, \mathrm{y}$ and $\mathrm{z}$ axes given as ii(x), i(y), i(z).Equation 22,23 and 24 are the source functions the three axes in dimensionless form.

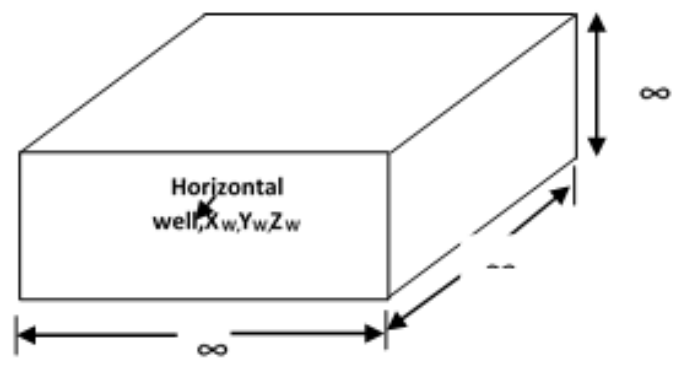

Fig 6: All boundaries are Infinite-acting

$$
\begin{aligned}
& S\left(x_{D}, t_{D}\right)=\frac{1}{2 \sqrt{\pi t_{D}}} \exp \left[-\frac{\left(x_{w D}-x_{D}\right)^{2}+\left(y_{w D}-y_{D}\right)^{2}}{4 t_{D}}\right] \\
& S\left(y_{D}, t_{D}\right)=\frac{1}{2 \sqrt{\pi t_{D}}} \exp \left[-\frac{\left(y_{w D}-y_{D}\right)^{2}}{4 t_{D}}\right] \\
& S\left(z_{D}, t_{D}\right)=\frac{1}{2 \sqrt{\pi t_{D}}} \exp \left[-\frac{\left(z_{w D}-z_{D}\right)^{2}}{4 t_{D}}\right]
\end{aligned}
$$

Putting equation 22,23 and 24 in 4 we have Equation 25 as the pressure distribution for a reservoir with all boundaries Infinite acting.

$$
P_{D}=2 \pi h_{D} \int_{0}^{t_{D}}\left\{\begin{array}{c}
\frac{1}{2 \sqrt{\pi t_{D}}} \exp \left[-\frac{\left(x_{w D}-x_{D}\right)^{2}+\left(y_{w D}-y_{D}\right)^{2}}{4 t_{D}}\right] \\
* \frac{1}{2 \sqrt{\pi t_{D}}} \exp \left[-\frac{\left(y_{w D}-y_{D}\right)^{2}}{4 t_{D}}\right] * \frac{1}{2 \sqrt{\pi t_{D}}} \exp \left[-\frac{\left(z_{w D}-z_{D}\right)^{2}}{4 t_{D}}\right]
\end{array}\right\} \partial t_{D}
$$

Water Edge and infinite acting

Figure 7 is a reservoir with water edge and Infinite acting. It has a horizontal well locations $x_{w}, y_{w}$ and $z_{w}$ in $x, y$ and $\mathrm{z}$ directions respectively. The source functions are selected from basic instantaneous source functions table for $\mathrm{x}, \mathrm{y}$ and $\mathrm{z}$ axis's given as $\mathrm{xi}(\mathrm{x}), \mathrm{i}(\mathrm{y}), \mathrm{i}(\mathrm{z})$.Equation 26,27 and 28 are the source functions the three axes in dimensionless form.

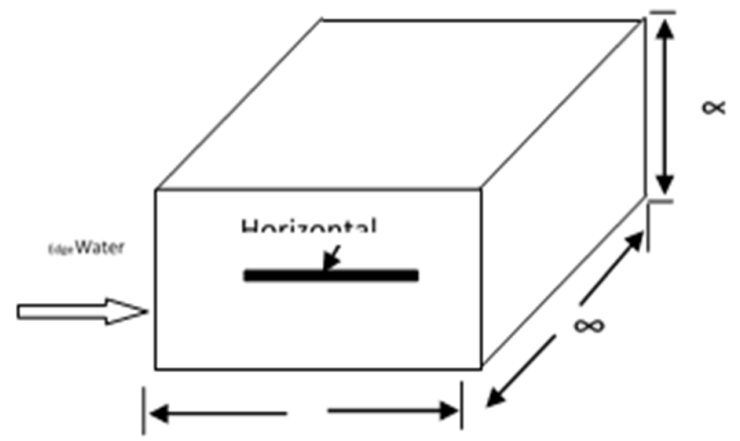

Fig 7: Reservoir with Water Edge and infinite-acting

$$
S\left(x_{D}, t_{D}\right)=\frac{8}{\pi} \sum_{n=1}^{\infty} \frac{1}{2 n-1} \exp \left[-\frac{(2 n-1)^{2} \pi^{2} t_{D}}{x_{e D}^{2}}\right] \cos \frac{(2 n-1) \pi x_{D}}{2 x_{e D}} \sin \frac{(2 n-1) \pi x_{D}}{x_{e D}} \sin \frac{(2 n-1) \pi x_{W D}}{x_{e D}}
$$


$S\left(y_{D}, t_{D}\right)=\frac{1}{2 \sqrt{\pi t_{D}}} \exp \left[-\frac{\left(y_{w D}-y_{D}\right)^{2}}{4 t_{D}}\right]$
$\left(z_{D}, t_{D}\right)=\frac{1}{2 \sqrt{\pi t_{D}}} \exp \left[-\frac{\left(z_{w D}-z_{D}\right)^{2}}{4 t_{D}}\right]$

Putting equation 26,27 and 28 in 4 we have Equation 29 as the pressure distribution for a reservoir with Water Edge and infinite acting.

$$
\begin{aligned}
P_{D}=\frac{8}{\pi} \sum_{\mathrm{n}=1}^{\infty} \frac{1}{2 \mathrm{n}-1} & \exp \left[-\frac{(2 \mathrm{n}-1)^{2} \pi^{2} \mathrm{t}_{\mathrm{D}}}{\mathrm{x}_{\mathrm{eD}}^{2}}\right] \cos \frac{(2 \mathrm{n}-1) \pi \mathrm{x}_{\mathrm{D}}}{2 \mathrm{x}_{\mathrm{eD}}} \sin \frac{(2 \mathrm{n}-1) \pi \mathrm{x}_{\mathrm{D}}}{\mathrm{x}_{\mathrm{eD}}} \sin \frac{(2 \mathrm{n}-1) \pi \mathrm{x}_{\mathrm{WD}}}{\mathrm{x}_{\mathrm{eD}}} \\
& * \frac{1}{2 \sqrt{\pi t_{D}}} \exp \left[-\frac{\left(y_{w D}-y_{D}\right)^{2}}{4 t_{D}}\right] * \frac{1}{2 \sqrt{\pi t_{D}}} \exp \left[-\frac{\left(z_{w D}-z_{D}\right)^{2}}{4 t_{D}}\right]
\end{aligned}
$$

Bottom water and all others infinite acting

Figure 8 is a reservoir with Bottom water Infinite acting. It has a horizontal well locations $x_{w}, y_{w}$ and $z_{w}$ in $x, y$ and $\mathrm{z}$ directions respectively. The source functions are selected from basic instantaneous source functions table for $\mathrm{x}, \mathrm{y}$ and $\mathrm{z}$ axis's given as $\mathrm{ii}(\mathrm{x}) \mathrm{i}(\mathrm{y})$ vii(z)equation 30,31 and 32 are the source functions the three axes in dimensionless form.

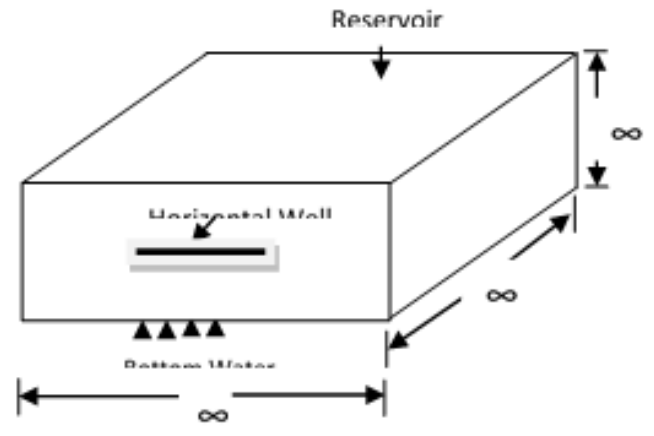

Fig 8: Bottom water and infinite acting

$$
\begin{aligned}
& S\left(x_{D}, t_{D}\right)=\frac{1}{2 \sqrt{\pi t_{D}}} \exp \left[-\frac{\left(x_{w D}-x_{D}\right)^{2}+\left(y_{w D}-y_{D}\right)^{2}}{4 t_{D}}\right] \\
& S\left(y_{D}, t_{D}\right)=\frac{1}{2 \sqrt{\pi t_{D}}} \exp \left[-\frac{\left.\left(y_{w D}-y_{D}\right)^{2}\right]}{4 t_{D}}\right] \\
& S\left(z_{D}, t_{D}\right)=\frac{2}{h_{D}} \sum_{n=1}^{\infty} \frac{1}{2 n-1} \exp \left[-\frac{(2 n-1)^{2} \pi^{2} t_{D}}{h_{D}^{2}}\right]_{\sin \frac{(2 n-1) \pi z_{W D}}{h_{D}}}
\end{aligned}
$$

Putting equation 30,31 and 32 in 4 we have Equation 33 as the pressure distribution for a reservoir with Bottom water and infinite acting.

$P_{D}=\frac{1}{2 \sqrt{\pi t_{D}}} \exp \left[-\frac{\left(x_{w D}-x_{D}\right)^{2}+\left(y_{w D}-y_{D}\right)^{2}}{4 t_{D}}\right] *$

$\frac{1}{2 \sqrt{\pi t_{D}}} \exp \left[-\frac{\left(y_{w D}-y_{D}\right)^{2}}{4 t_{D}}\right] * \frac{1}{2 n-1} \exp \left[-\frac{(2 n-1)^{2} \pi^{2} t_{D}}{h_{D}{ }^{2}}\right] *$

$\sin \frac{(2 n-1) \pi z_{D}}{h_{D}} * \sin \frac{(2 n-1) \pi z_{W D}}{h_{D}}$ 


\section{Top Gas Cap and infinite Acting}

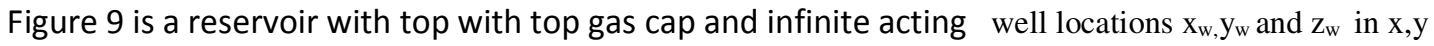
and $z$ directions respectively. The source functions are selected from basic instantaneous source functions table for $\mathrm{x}, \mathrm{y}$ and $\mathrm{z}$ axis's given as ii(x) $\mathrm{i}(\mathrm{y})$ vi(z). Equation 34,35 and 36 are the source functions the three axes in dimensionless form.

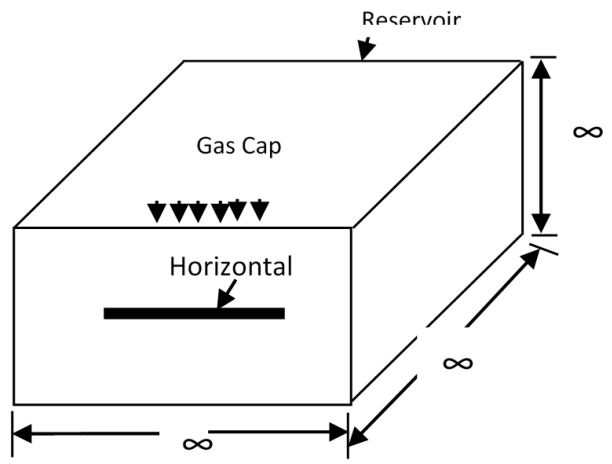

Fig 9: Reservoir Top Gas Cap and Infinite acting

$$
\begin{gathered}
S\left(x_{D}, t_{D}\right)=\frac{1}{2 \sqrt{\pi t_{D}}} \exp \left[-\frac{\left(x_{w D}-x_{D}\right)^{2}+\left(y_{w D}-y_{D}\right)^{2}}{4 t_{D}}\right] \\
S\left(y_{D}, t_{D}\right)=\frac{1}{2 \sqrt{\pi t_{D}}} \exp \left[-\frac{\left(y_{w D}-y_{D}\right)^{2}}{4 t_{D}}\right] \\
S\left(z_{D}, t_{D}\right)=\frac{1}{h_{D}}\left\{1+2 \sum_{n=1}^{\infty} \exp \left[-\frac{l^{2} \pi^{2} t_{D}}{h_{D}}\right] \sin \frac{l \pi z_{D}}{h_{D}} \sin \frac{l \pi z_{w D}}{h_{D}}\right\}
\end{gathered}
$$

Putting equation 34,35 and 36 in 4 we have Equation 37 as the pressure distribution for a reservoir with Bottom water and infinite acting.

$P_{D}=\frac{1}{2 \sqrt{\pi t_{D}}} \exp \left[-\frac{\left(x_{w D}-x_{D}\right)^{2}+\left(y_{w D}-y_{D}\right)^{2}}{4 t_{D}}\right] * \frac{1}{2 \sqrt{\pi t_{D}}} \exp \left[-\frac{\left(y_{w D}-y_{D}\right)^{2}}{4 t_{D}}\right]$
$* \frac{1}{h_{D}}\left\{1+2 \sum_{n=1}^{\infty} \exp \left[-\frac{l^{2} \pi^{2} t_{D}}{h_{D}}\right] \sin \frac{l \pi z_{D}}{h_{D}} \sin \frac{l \pi z_{w D}}{h_{D}}\right\}$

Infinite at $z$ axis and completely sealed at $x$ axis and $y$ axis: Figure 10 is a reservoir with Infinite at $\mathrm{z}$ axis and completely sealed at $\mathrm{x}$ axis and $\mathrm{y}$-axis. It has a horizontal well locations at $\mathrm{x}_{\mathrm{w}}, \mathrm{y}_{\mathrm{w}}$ and $\mathrm{z}_{\mathrm{w}}$ in $\mathrm{x}, \mathrm{y}$ and $\mathrm{z}$ directions respectively. The source functions are selected from basic instantaneous source functions table for $\mathrm{x}, \mathrm{y}$ and $\mathrm{z}$ axis's given as viii(x), $\mathrm{v}(\mathrm{y})$, i(z). Equation 38, 39 and 40 are the source functions in dimensionless form.

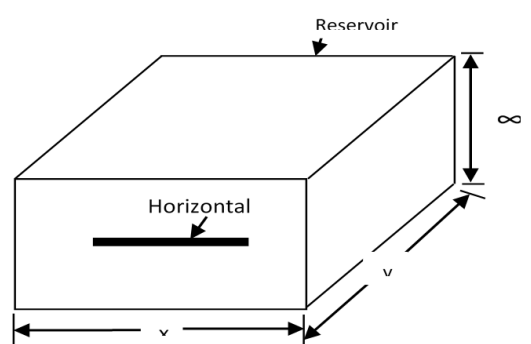

Fig 10: Infinite at $\mathrm{z}$ axis and completely sealed at $\mathrm{x}$ axis and $\mathrm{y}$-axis

$$
S\left(x_{D}, t_{D}\right)=\frac{2}{X_{e D}}\left\{1+\frac{2 X_{e D}}{\pi} \sum_{n=1}^{\infty} \frac{1}{n} \exp \left[-\frac{n^{2} X_{D}^{2} t_{D}}{X_{e D}{ }^{2}}\right] \sin \frac{n \pi}{x_{e D}} \cos \frac{n \pi x_{w D}}{X_{e D}} \cos \frac{n \pi x_{D}}{X_{e D}}\right\}
$$




$$
\begin{gathered}
S\left(y_{D}, t_{D}\right)=\frac{1}{y_{D}}\left\{1+2 \sum_{n=1}^{\infty} \exp \left[-\frac{m^{2} \pi^{2} t_{D}}{y_{D}}\right] \cos \frac{m \pi y_{w D}}{y_{D}} \cos \frac{m \pi y_{D}}{y_{D}}\right\} \\
S\left(z_{D}, t_{D}\right)=\frac{1}{2 \sqrt{\pi t_{D}}} \exp \left[-\frac{\left(z_{w D}-z_{D}\right)^{2}}{4 t_{D}}\right]
\end{gathered}
$$

Putting equation38,39 and 40 in 4 we have Equation 41 as the pressure distribution for a Reservoir with Infinite at $\mathrm{z}$ axis and completely sealed at $\mathrm{x}$ axis and $\mathrm{y}$-axis

$$
\begin{gathered}
P_{D}=\frac{2}{\mathrm{X}_{\mathrm{eD}}}\left\{1+\frac{2 \mathrm{X}_{\mathrm{eD}}}{\pi} \sum_{\mathrm{n}=1}^{\infty} \frac{1}{\mathrm{n}} \exp \left[-\frac{\left.\left.\mathrm{n}^{2} \mathrm{X}_{\mathrm{D}}{ }^{2} \mathrm{t}_{\mathrm{D}}\right] \sin \frac{\mathrm{n} \pi}{\mathrm{X}_{\mathrm{eD}}{ }^{2}} \cos \frac{\mathrm{n} \pi \mathrm{x}_{\mathrm{wD}}}{\mathrm{X}_{\mathrm{eD}}} \cos \frac{\mathrm{n} \pi \mathrm{x}_{\mathrm{D}}}{\mathrm{X}_{\mathrm{eD}}}\right\} *}{\frac{1}{\mathrm{y}_{\mathrm{D}}}\left\{1+2 \sum_{\mathrm{n}=1}^{\infty} \exp \left[-\frac{\mathrm{m}^{2} \pi^{2} \mathrm{t}_{\mathrm{D}}}{\mathrm{y}_{\mathrm{D}}}\right] \cos \frac{\mathrm{m} \pi \mathrm{y}_{\mathrm{wD}}}{\mathrm{y}_{\mathrm{D}}} \cos \frac{\mathrm{m} \pi \mathrm{y}_{\mathrm{D}}}{\mathrm{y}_{\mathrm{D}}}\right\} *}\right.\right. \\
\frac{1}{2 \sqrt{\pi t_{D}}} \exp \left[-\frac{\left(z_{w D}-z_{D}\right)^{2}}{4 t_{D}}\right]
\end{gathered}
$$

\section{RESULTS AND DISCUSION}

The derivation of ten (10) Pressure distribution models for horizontal well using source function has been carried out and the results are presented as follow:

Completely Bounded Reservoir: The derived model for Pressure distribution for completely bounded reservoir shown in fig. 1 is given as eq. 5. Along the $\mathrm{x}$-axis, this model has infinite-slab sources in infiniteslab sources as shown in eq.1, along the y-axis it has infinite-plane sources in infinite-slab sources as shown in eq.2. Along z-axis, it has infinite-plane sources in infinite-slab sources as shown in eq.3, only $x$-axis is sealed: The derived model for Pressure distribution for Reservoir with only $x$-axis sealed shown in fig. 2 is given as eq.9. Along the $\mathrm{x}$-axis, this model has infinite-slab sources in infinite-slab sources as shown in eq.6.Along the y- axis it has infinite sources as shown in eq.7. Along z-axis, it has infinite sources as shown in eq.8. Reservoir subject to Edge water and sealed at $\mathrm{y}$ and $\mathrm{z}$ axes. The derived model for Pressure distribution for Reservoir subject to Edge water and sealed at $\mathrm{y}$ and $\mathrm{z}$ axes shown in fig. 3 is given in eq.13.Along the $\mathrm{x}$-axis, it has infinite-slab sources in infinite-slab sources as shown in eq.10, along the yaxis it has infinite-plane sources in infinite-slab sources as shown in eq.11and along the z-axis, it has infinite-plane sources in infinite-slab sources as shown in eq.12. Bottom water and sealed at $x$ and y axes: The derived model for Pressure distribution for Reservoir subject to Bottom water and sealed at $\mathrm{x}$ and $\mathrm{y}$ axes shown in fig.4 is given in eq.17. Along the $\mathrm{x}$-axis, it has infinite-slab sources in infinite-slab sources as shown in eq.14, along the $\mathrm{y}$-axis it has infinite-plane sources in infinite-slab sources as shown in eq.15. Top Gas Cap and Bottom Water drive: The derived model for Pressure distribution for Reservoir subject Top Gas Cap and Bottom Water drive shown in fig.5 is given in eq.21.Along the x-axis, it has infinite-slab sources in infinite-slab sources as shown in eq.18, along the y- axis it has infinite-plane sources in infinite-slab sources as shown in eqs19 and along z-axis, it has infinite-plane sources in infinite-slab sources as shown in eq. 20

All boundaries are Infinite acting: The derived model for Pressure distribution for Reservoir with all boundaries been Infinite acting shown in fig. 6 is given in eq.25.Along the axis it has infinite sources as shown in eq. 22 , along the $y$ - axis it has infinite sources shown in eq. 23 and along the $\mathrm{z}$ - axis it has infinite sources shown in eq. 24

Water Edge and Infinite acting:

The derived model for Pressure distribution for reservoir subjected to water edge and infinite acting shown in fig. 7 is given in eq.29. Along the $\mathrm{x}$-axis, it has infinite-slab sources in infinite-slab sources as shown in eq.26, along the $y$ - axis it has infinite sources as shown in eq. 27 and along $\mathrm{z}$-axis, and it has infinite sources as shown in eq. 28 .

. Bottom water and all others infinite acting: The derived model for Pressure distribution for reservoir subjected to bottom water and infinite acting shown in fig. 8 is given in eq.33. Along the $\mathrm{x}$-axis it has infinite sources as shown in eq.30, along the $y$ - axis it has infinite sources as shown in eq. 31 and along z-axis it has infinite-plane sources in infinite-slab sources as shown in eq.32.

Gas cap and all others infinite acting: The derived model for Pressure distribution for reservoir subjected to Gas cap and infinite acting shown in fig. 9 is given in eq.37. Along the $\mathrm{x}$-axis it has infinite sources as shown in eq.34, along the y-axis it has infinite as shown in eq. 35 and along $\mathrm{z}$-axis, it has infinite-plane sources in infinite-slab sources as shown in eq.36. Infinite at $\mathrm{z}$ axis and completely sealed at $\mathrm{x}$ axis and $\mathrm{y}-$ axis. 
The derived model for Pressure distribution for reservoir with. Infinite at $\mathrm{z}$ axis and completely sealed at $\mathrm{x}$ axis and $\mathrm{y}$-axis shown in fig. 10 is given in eq.41. Along the $\mathrm{x}$-axis, it has infinite-slab sources in infinite-slab sources as shown in eq.38, along the yaxis they have infinite-plane sources in infinite-slab sources as shown in eq. 39 and along $\mathrm{z}$-axis it has infinite sources as shown in eq. 40 .

Conclusion: The following observations about the source functions are: (i) three instantaneous source functions are involved in the expression. (ii) for along the $\mathrm{x}$-axis, seven of the models have infinite-slab sources in infinite-slab sources.

\section{REFERENCES}

Adewole, ES; Olafuyi, OA (2010). "The use of source and Green's Function to derive dimensionless pressure and derivative distribution of A twolayered reservoir Part 1: A- Architecture", Journal of Mathematics and Technology, April, 2010, pp.92-101.
Chawla, A; Kirby JT (2000). A source function method for generation of waves on currents in Boussinesq models, Applied Ocean Research 22 (2000) 75-83.

Eiroboyi I; Wilkie, SI (2017). Comparative Evaluation of Pressure Distribution Between Horizontal And Vertical Wells In A Reservoir (Edge Water Drive), Nigerian Journal of Technology (NIJOTECH)Vol. 36, No. 2, April 2017, pp. 457 460.

Gringarten, AC; Ramey, HJ (1973). "The Use of Source and Green's Functions in Solving Unsteady-Flow Problems in Reservoirs," SPEJ, pp. $255-285$, Oct. 1973 .

1. Jacob, O (2016). Expanding the usage of the Source Function to experimental electron, densities, Structural Science Crystal Engineering Materials, 17 March 2016.

2. Larry, WL (2006).Petroleum Engineering Handbook Vol.1, pp.1-100.

3. Ohaegbulam, MC; Izuwa, NC; Onwukwe, SI (2017). Analysis of Wellbore Pressure Drop on Horizontal Well Performance, Oil Gas Research, 2017, 3:2. 